\title{
Physical Activity and Body Composition Relate to Cardiorespiratory Fitness Independently in Young Adults with Down Syndrome
}

\author{
Shannon D. R. Ringenbach ${ }^{1}$, Simon D. Holzapfel ${ }^{1}$, Pamela R. Bosch ${ }^{2}$, Lauren M. Hunt ${ }^{1}$ and Michelle Snow ${ }^{1}$ \\ 1. Department of Exercise Science and Health Promotion, Arizona State University, Phoenix, AZ 85004, USA \\ 2. Department of Physical Therapy, Northern Arizona University, Phoenix, AZ 85004, USA
}

\begin{abstract}
BACKGROUND: Persons with Down syndrome (DS) typically possess low cardiorespiratory fitness (CRF), engage in low levels of physical activity (PA), and display elevated obesity levels, but the association between these variables is not clear in persons with DS. METHOD: We investigated the relationships among measures of CRF, PA, and body composition in 30 young adults with DS (age: $21.3 \pm 5.5$ years) using a cross-sectional study design. RESULTS: Male participants presented on average with a BMI of $32.9 \pm$ $10.0 \mathrm{~kg} / \mathrm{m}^{2}$, a percent body fat of $22.2 \pm 13.0 \%$, and a six-minute walk distance (6MWD) of $411.7 \pm 66.3 \mathrm{~m}$. Female participants presented on average with a BMI of $31.6 \pm 13.5 \mathrm{~kg} / \mathrm{m}^{2}$, a percent body fat of $26.7 \pm 15.1 \%$, and a six-minute walk distance (6MWD) of $360.4 \pm 91.2 \mathrm{~m}$. $6 \mathrm{MWD}$ shared a positive relationship with leisure time physical activity levels $(r=0.56, \beta=1.488)$ and a negative relationship with body mass index (BMI; $r=-0.49, \beta=-2.561$ ). No relationship between body composition and PA was found. CONCLUSIONS: It seems that higher PA levels and lower BMI relate to better CRF in young adults with DS. Physical activity and body composition appear to be unrelated in persons with DS.
\end{abstract}

Key words: Intellectual disability, six minute walk test, percent body fat, body mass index.

\author{
Abbreviations \\ $\% \mathrm{BF}=$ percent body fat \\ $\mathrm{BMI}=$ body mass index \\ $\mathrm{CA}=$ chronological age \\ $\mathrm{CRF}=$ cardiorespiratory fitness \\ DEXA = dual-energy X-ray absorptiometry \\ $\mathrm{DS}=$ Down syndrome \\ ID = intellectual disability \\ LTPA = leisure time physical activity \\ $\mathrm{MA}=$ mental age \\ $\mathrm{PA}=$ physical activity
}

\section{Introduction}

Persons with Down syndrome (DS) are commonly referred to as an at risk population due to poor health and cardiorespiratory fitness (CRF) levels [1]. The peak aerobic capacity is reported to be lower in individuals with DS than individuals with other

Corresponding author: Simon D. Holzapfel, Ph.Dc., clinical assistant professor, research field: exercise physiology and exercise psychology. intellectual disabilities (IDs) or individuals without IDs [2]. Low CRF is associated with excess cardiovascular disease and mortality risk [3-7]. Additionally, low fitness levels in individuals with DS have been associated with impaired performance on functional tasks of daily living such as rising from a chair, walking speed, and ascending and descending stairs [8]. CRF also shares a positive relationship with cognitive function in the general population $[9,10]$. Both functional tasks of daily living and cognitive function are areas of weakness in individuals with DS [11-13]. However, very few exercise intervention studies were successful in improving CRF in individuals with DS [14-18]. It is important to investigate correlates and potential modifiers of CRF in persons with DS.

\subsection{Body Composition and Cardiorespiratory Fitness}

Excess adiposity is associated with increased disease incidence and mortality in the general population [19], 
and the prevalence of obesity and associated morbidities such as cardiovascular disease have also been shown to be elevated among children and adolescents with DS [16]. Obesity has also been shown to be negatively associated with $\mathrm{CRF}$ (i.e. $\mathrm{VO}_{2}$ peak) in persons with DS [2]. BMI has also been negatively correlated with the six minute walk distance (6MWD; $r$ $=-0.30)$ in a sample of adolescents with IDs including DS [20]. Nordstrøm and colleagues [21] reported a difference in 6MWD of $111 \mathrm{~m}$ between obese and normal or underweight Norwegian adults who had either DS, Prader-Willi syndrome, or Williams syndrome. The results of these studies provide general evidence of the association between body composition and CRF in persons with IDs, but findings that pertain specifically to populations with DS continue to be sparse [2].

\subsection{Physical Activity and Cardiorespiratory Fitness}

Matute-Llorente and colleagues [22] reported that adolescents with DS spent less time in all intensities of physical activity (PA) than typically-developing peers. Others reported that less than one third of people with IDs, including DS, engage in at least 30 minutes of moderate to vigorous activity on most days of the week [23]. Nordstrøm and colleagues [21] also report low levels of PA and large amounts of sedentary time in persons with DS. Draheim et al. [24] reported that $45 \%$ of adults with IDs meet the recommended 150 minutes of moderate to vigorous PA per week.

Despite the known association between greater leisure time PA (LTPA) levels and improved CRF in the general population [25], this relationship has only once been investigated in populations with DS. Balic and colleagues [26] reported greater CRF in individuals with DS who participate in Special Olympics compared to those that do not. However, a major limitation of that study was the dichotomous nature in which LTPA was defined (Special Olympics participation vs. no Special Olympics participation) which does not allow us to quantify the amount of
LTPA that is associated with better CRF. Even though Nordstrøm et al. [21] had objectively measured data on LTPA (accelerometry) and CRF (6MWD) in adults with DS, they did not examine the relationship between those variables. To date, most evidence for a relationship between PA and CRF in DS comes from intervention studies [27, 28]. Despite these studies, observational investigations are needed to determine the association between free-living LTPA and CRF in individuals with DS.

\subsection{Physical Activity and Body Composition}

The association between LTPA and body composition in persons with DS is not clear because the relationship between PA and body composition in this population has only been studied in the context of targeted exercise intervention. Combined cardiovascular and strength training did not improve body weight, skinfold measures or BMI [29], while plyometric jump training increased lean mass without a change in percent body fat [16], and aerobic training both decreased fat mass and increased lean mass [30].

Some evidence concerning the relationship between LTPA and body composition comes from [21]. They found no relationship between total PA and BMI in a sample of Norwegian adults that included DS, Prader-Willi syndrome, and Williams syndrome. They did find, however, that obese individuals spent 10 minutes less in moderate to vigorous PA per day than individuals in lower BMI categories. Since their sample included individuals with other genetic disorders, the relationship between LTPA and body composition in persons with DS remains unclear, and they did not include a direct measure of body composition such as percent body fat $(\% \mathrm{BF})$.

\subsection{The Current Study}

Predictors of CRF, namely LTPA and body composition, and their relationship to CRF specifically in persons with DS needs to be explored. Therefore, we sought to examine the relationship among LTPA, 
measures of body composition and anthropometry, and CRF in young adults with DS using measures that are easily administered in field or clinical settings. LTPA as opposed to other measures of PA, such as total physical activity, was assessed because it is commonly the subject of studies involving PA in persons with DS [24, 26, 31-33] and because occupational physical activity is most likely low amongst persons with DS [34-36]. The peak distance a person is able to walk in six minutes (i.e. 6MWD) is often used as a practical and easily administered assessment of CRF among compromised populations and it was therefore used in the current study [37-39]. The main purpose of this study was to determine the association of body composition and LTPA levels with 6MWD in persons with DS. A secondary aim was to investigate the relationship between LTPA levels and body composition.

\subsection{Hypotheses}

More favorable values of anthropometry and body composition (BMI, waist circumference [WC], and percent body fat $[\% \mathrm{BF}]$ ) are associated with greater CRF.

Greater amounts of LTPA are associated with greater CRF.

Greater amounts of LTPA are associated with more favorable body composition.

\section{Methods}

\subsection{Participants}

Thirty young adults with trisomy-21 DS (22 male, 8 female) who lived with their families participated in this study (participant characteristics located in Table 1). Parents or guardians confirmed the diagnosis of trisomy-21 during screening. Participants were recruited at local events specific to persons with IDs, local organizations providing recreational opportunities (e.g. Best Buddies, Special Olympics, municipal parks and recreation departments), flyer distribution via listservs of local organizations, and through word of mouth. The study protocol was approved by the human subjects institutional review board of the university.

\subsection{Procedure}

All procedures took place in a controlled laboratory environment. First, the participants and parents or guardians were familiarised with the lab and all testing procedures. The participants then read (or were read to) and signed assent forms while their parents/guardians read and signed consent forms before data collection began. Consent, assent, and participation were voluntary and it was made clear that participation could be discontinued at any time. First, mental age (MA) was assessed with the Peabody Picture Vocabulary

Table 1 Descriptive statistics of the entire sample (combined) and by gender.

\begin{tabular}{|c|c|c|c|c|c|c|c|}
\hline \multirow[b]{2}{*}{ Variable } & \multicolumn{2}{|c|}{ Combined $(n=30)$} & \multicolumn{2}{|c|}{ Male $(n=22)$} & \multicolumn{2}{|c|}{ Female $(n=8)$} & \multirow{2}{*}{-Gender diff. ( $P$-value $)^{*}$} \\
\hline & Mean & SD & Mean & SD & Mean & SD & \\
\hline CA (years) & 21.26 & 5.46 & 21.09 & 5.36 & 21.72 & 6.07 & 0.629 \\
\hline MA (years) & 6.04 & 1.80 & 5.79 & 1.84 & 6.71 & 1.61 & 0.087 \\
\hline Weight $(\mathrm{kg})$ & 73.63 & 23.47 & 75.47 & 22.86 & 68.56 & 25.97 & 0.368 \\
\hline BMI $\left(\mathrm{kg} / \mathrm{m}^{2}\right)$ & 32.54 & 10.78 & 32.88 & 9.96 & 31.61 & 13.51 & 0.730 \\
\hline$\% \mathrm{BF}$ & 23.40 & 13.44 & 22.20 & 12.95 & 26.71 & 15.10 & 0.565 \\
\hline $\mathrm{WC}(\mathrm{cm})$ & 92.89 & 26.11 & 92.20 & 27.48 & 94.81 & 23.52 & 0.872 \\
\hline LTPA score & 34.97 & 26.28 & 38.36 & 28.19 & 25.63 & 18.44 & 0.298 \\
\hline 6MWD (m) & 397.98 & 75.67 & 411.66 & 66.33 & 360.36 & 91.20 & 0.118 \\
\hline
\end{tabular}

Note. Abbreviations: $\mathrm{SD}=$ standard deviation, $\mathrm{CA}=$ chronological age, $\mathrm{MA}=$ mental age, $\mathrm{BMI}=$ body mass index, $\% \mathrm{BF}=$ percent body fat, $\mathrm{WC}=$ waist circumference, $\mathrm{LTPA}=$ leisure time physical activity, $6 \mathrm{MWD}=6$ minute walk distance.

*Mann-Whitney U Test. 
Test 4 (PPVT-4; [40]) which is a test of receptive language that has been widely used in populations with IDs. A researcher who was trained according to standard testing procedures [40] administered the PPVT-4.

\subsubsection{Body Composition and Anthropometry}

A series of assessments followed, including measurement of height $(\mathrm{cm})$ and weight $(\mathrm{kg})$ from which BMI $\left(\mathrm{kg} / \mathrm{m}^{2}\right)$ was subsequently calculated, WC $(\mathrm{cm})$, and \%BF. WC was measured around the narrowest part of the torso between the navel and chest, when seen from the front, and after the participant had completely exhaled. \%BF was measured with a bipedal bioelectrical impedance scale (Omron BF-400; Omron Medizintechnik, Mannheim, Germany). The \%BF measured with the Omron BF-400 has been shown to agree $(r=0.94)$ with dual-energy X-ray absorptiometry (DEXA) and it is not biased relative to DEXA measurements in regards to lower or higher $\% \mathrm{BF}$ even when hydration status is not controlled [41]. \%BF values determined with bioelectrical impedance measurements have also been validated against DEXA measurements in adolescents with DS $(\mathrm{r} \approx 0.90$; [42]). On average, the Omron BF-400 underestimated \%BF by $0.48 \%$ (95\% confidence interval: $\pm 6.62 \%)$ and the device was recommended for group estimates in research settings across BMI values ranging from underweight to obese [41].

\subsubsection{Leisure Time Physical Activity}

The parent or guardian of each participant completed the Godin LTPA survey [43] to assess the amount of LTPA their child or dependent completed during a typical week. The Godin LTPA survey required the parent/guardian to report how many times per week the participant engaged in bouts of at least 15 minutes in duration of mild, moderate, or vigorous PA. The number of vigorous bouts was weighted by a factor of 9 , the moderate bouts by a factor of 5 , and the mild bouts by a factor of 3. The weighting factors roughly correspond with metabolic equivalent (MET) values [44]. The weighted frequencies were summed to obtain the LTPA score used for the analyses. This score represents an estimate of MET-minutes/week if multiplied by 15 . For instance, a participant who engages in 5 bouts of mild PA, 3 bouts of moderate PA, and 2 bouts of vigorous PA per week would have a weighted score of 48. This would equal 720 MET-minutes/week. The Godin LTPA survey has been shown to correlate with other activity rating scales and with daily energy expenditure and it has adequate to excellent test-retest reliability $(I C C=0.62-0.81$; [43]).

\subsubsection{Cardiorespiratory Fitness}

The CRF of each participant was assessed with the 6MWD (m). Test-retest reliability of the 6MWD has been established for adolescents and young adults with DS [45]. The 6MWD has been found to be a valid and reliable proxy of relative $\mathrm{VO}_{2}$ peak (i.e. CRF) in adolescents, adults, and seniors with IDs including DS $[20,46,47]$ and it is a reliable measure in adults with DS (ICC $=0.93$; [48]). The 6MWD may also better reflect everyday functional capacity than calorimetry through oxygen consumption [49]. Additionally, poor 6MWD has been associated with increased mortality risk in patients with cardiopulmonary disease [37]. The six minute walk test was conducted by a single trained researcher in accordance with the guidelines by the American Thoracic Society (ATS; [49]) and other studies which also followed the ATS guidelines [20, 21]. To measure $6 \mathrm{MWD}$, participants walked as many laps as they could around two orange cones placed $25 \mathrm{ft}$. apart in a straight corridor. The participants were instructed to walk as far as they could in 6 minutes. In order to familiarise the participant with the test, the researcher walked two laps while the participant stood and watched. During the test, the researcher marked each completed lap on a data collection sheet. The researcher did not walk with the participant during the test but provided standard verbal encouragement and positive feedback throughout the test. After 6 minutes, the researcher told the participant to stop in place and the remaining distance from the starting cone was measured to the nearest meter and added to the total 
distance walked.

\subsection{Statistical Analyses}

Gender differences in all variables were tested using the Mann-Whitney U Test (Table 1). Two-tailed, bivariate correlations were computed using Pearson product-moment correlation coefficients. A correlation matrix of all study variables is displayed in Table 2 .

Multiple stepwise and hierarchical regression analyses with 6MWD as the dependent variable were completed. BMI, WC, \%BF, and LTPA score were used as predictor variables. Raw scores of BMI were retained and used in the regression analyses, as BMI shared the strongest correlation with 6MWD out of all the anthropometric and body composition variables (Table 2). However, BMI correlated strongly with WC (0.732) and \%BF (0.766). To avoid issues of multicollinearity, $\mathrm{WC}$ and $\% \mathrm{BF}$ were regressed on $\mathrm{BMI}$ and the unstandardised residuals $\left(\mathrm{WC}_{\mathrm{res}}\right.$ and $\% \mathrm{BF}_{\text {res }}$ ) were saved and used as predictor variables in the models. The residuals contain information about $\mathrm{WC}$ and $\% \mathrm{BF}$ that is not linearly predictable from BMI. Thus, $\mathrm{WC}_{\text {res }}$ and $\% \mathrm{BF}_{\text {res }}$ scores represented information about $\mathrm{WC}$ and $\% \mathrm{BF}$ that was not contained in BMI and they were completely orthogonal to BMI, which eliminated any chance of multicollinearity. $\mathrm{WC}_{\text {res }}$ and $\% \mathrm{BF}_{\text {res }}$ scores were entered into the stepwise regression models to find out if they contained unique information about 6MWD performance that was not predicted by BMI. First, all variables (BMI,
$\mathrm{WC}_{\text {res }}, \% \mathrm{BF}_{\text {res }}$, and LTPA) were entered into a stepwise regression model. Next, a hierarchical regression was used to control for the effects of chronological age (CA) on $6 \mathrm{MWD}$, because $\mathrm{CA}$ correlated significantly with 6MWD and all predictor variables (Table 2).

\section{Results}

Descriptive statistics are summarised in Table 1. There were no significant differences between male and female participants in any variable. The correlations among all variables are presented in Table 2. Correlations of $6 \mathrm{MWD}$ to CA, BMI, and LTPA were significant. There were no significant correlations between LTPA scores and measures of adiposity or anthropometry.

Preliminary analyses indicated that the assumptions of normality, linearity, multicollinearity, and homoscedasticity were not violated. Only LTPA ( $\beta=$ 1.393, $S E=0.497, P=0.009)$ and BMI $(\beta=-2.222, S E$ $=1.017, P=0.038$ ) entered the model as significant predictors of $6 \mathrm{MWD}$. The model explained $41 \%$ of the variance in 6MWD before adjusting for CA (intercept $=428.5 ; F(2,27)=9.45, P=0.001)$ and $27 \%$ after adjusting for $\mathrm{CA}\left(\beta_{\mathrm{CA}}=1.216, S E=2.645, P=0.650\right.$; $\beta_{\mathrm{LTPA}}=1.488, S E=0.546, P=0.011 ; \beta_{\mathrm{BMI}}=-2.561, S E$ $=1.268, P=0.054$; intercept $=410.4, F(3,26)=6.19, P$ $=0.003 ; F$ change $(2,26)=5.94, P=0.007)$. While CA explained a significant amount of variance on its own $\left(15 \% ; \beta_{\mathrm{CA}}=-4.842, P=0.035\right.$; intercept $=505.7$, $F(1,28)=4.93, P=0.035)$ and significantly changed

Table 2 Correlation matrix.

\begin{tabular}{llllllll}
\hline & $6 \mathrm{MWD}$ & LTPA & WC & $\%$ BF & BMI & Weight & MA \\
\hline CA & $-0.387^{*}$ & $-0.491^{* *}$ & $0.558^{* *}$ & $0.503^{* *}$ & $0.642^{* *}$ & $0.573^{* *}$ & 0.189 \\
MA & 0.160 & 0.140 & -0.024 & -0.028 & 0.026 & 0.650 & - \\
Weight & -0.354 & -0.232 & $0.728^{* *}$ & $0.642^{* *}$ & $0.834^{* *}$ & - & \\
BMI & $-0.490^{* *}$ & -0.337 & $0.732^{* *}$ & $0.766^{* *}$ & - & & \\
\%BF & -0.272 & -0.286 & $0.656^{* *}$ & - & & & \\
WC & -0.359 & -0.344 & - & & & & \\
LTPA & $0.555^{* *}$ & - & & & & \\
6MWD & - & & & & & & \\
\hline
\end{tabular}

Note. Abbreviations: $\mathrm{CA}=$ chronological age, $\mathrm{MA}=$ mental age, $\mathrm{BMI}=$ body mass index, $\% \mathrm{BF}=$ percent body fat, $\mathrm{WC}=$ waist circumference, $\mathrm{LTPA}=$ leisure time physical activity, $6 \mathrm{MWD}=6$ minute walk distance.

$* P<0.05 ; * * P<0.001$. 
the model, LTPA and BMI remained significant predictors after adjustment.

\section{Discussion}

To our knowledge, this research is the first to examine the relationship of an estimate of aerobic capacity (6MWD) to anthropometric and LTPA measures specifically in persons with DS. We found that BMI and LTPA were the strongest predictors of CRF in young adults with DS, but BMI and LTPA were not related.

\subsection{Sample Characteristics}

Walking a distance of less than $350 \mathrm{~m}$ in six minutes has been associated with increased risk for mortality, chronic heart failure, and pulmonary arterial hypertension [37]. Our participants who had a 6 MWD in the fourth quartile walked an average of $322 \mathrm{~m}$. Six of these participants walked less than $350 \mathrm{~m}$ suggesting that $20 \%$ of our sample may be at a significantly elevated cardiovascular disease and mortality risk. Newman and colleagues [50] found that the inability to complete a $400 \mathrm{~m}$ walk is associated with an $86 \%$ increased risk for cardiovascular disease and mortality and a $95 \%$ increased risk for mobility disability. They also reported that each additional minute it took to complete the $400 \mathrm{~m}$ walk was associated with a $29 \%$ increase in mortality risk, a $20 \%$ increase in cardiovascular disease risk, and a 52\% increased risk for mobility limitations and disability. In our sample, this would translate to a mean increased risk of approximately $71 \%, 49 \%$, and $127 \%$ for mortality, cardiovascular disease incidence, and mobility limitations/disability, respectively, from the lowest to the highest 6MWD quartile. In the study by Newman et al. [50], those who completed the $400 \mathrm{~m}$ in $>362$ seconds had a $223 \%$ higher mortality risk than those who completed it in $<290$ seconds. In our sample, $37 \%$ took more than 362 seconds when we extrapolated the $6 \mathrm{MWD}$ to $400 \mathrm{~m}$. None of the participants would have completed $400 \mathrm{~m}$ in less than 290 seconds based on their distance covered in 6 minutes.

In accordance with other studies [51,52], we found that the prevalence of obesity in our sample was at least as high as or higher than the prevalence in the general population. Overall, $50 \%$ of participants were obese based on \%BF [53]. Among female participants, 37.5\% were obese as defined by a $\% \mathrm{BF}$ over $30 \%$ [53]. Among male participants, $54.5 \%$ were obese with a $\%$ BF over $25 \%$ [53].

The PA recommendations by the US Surgeon General equate to 14 LTPA points on the Godin questionnaire [44]. Eighty percent of our sample met this recommendation when light PA was included in the calculations. However, only $30 \%$ of participants met the recommendation of moderate to vigorous PA for at least 150 minutes per week. Draheim et al. [24] reported that $45 \%$ of participants met these recommendations. However, the study by Draheim et al. [24] included individuals with non-DS IDs. Vigorous PA for 75 minutes per week was reported for only one (3\%) participant in our study, similar to the prevalence reported by Draheim et al. [24].

\subsection{Body Composition and Cardiorespiratory Fitness}

The results of our study support our hypothesis that anthropometric measures are associated with CRF. Measures of body composition related to CRF in the expected direction in young adults with DS. For example, 6MWD increased as BMI decreased (Fig. 1). Interestingly, the greatest distance was achieved by those in the 24.0 to $31.3 \mathrm{BMI}$ range, which includes the BMI range that is conventionally considered "overweight". It has been shown that all-cause mortality is lowest in this range of BMI in the general population [54]. The difference in 6MWD between the first (>38.3) and third quartile (24-31.3) of BMI was $64 \mathrm{~m}$, a difference that is considered clinically important [37, 55] and greater than the minimal detectable change (approximately $50 \mathrm{~m}$ ) in adults with DS [48]. In older adults, stroke survivors, and persons with chronic obstructive pulmonary disease, a difference 


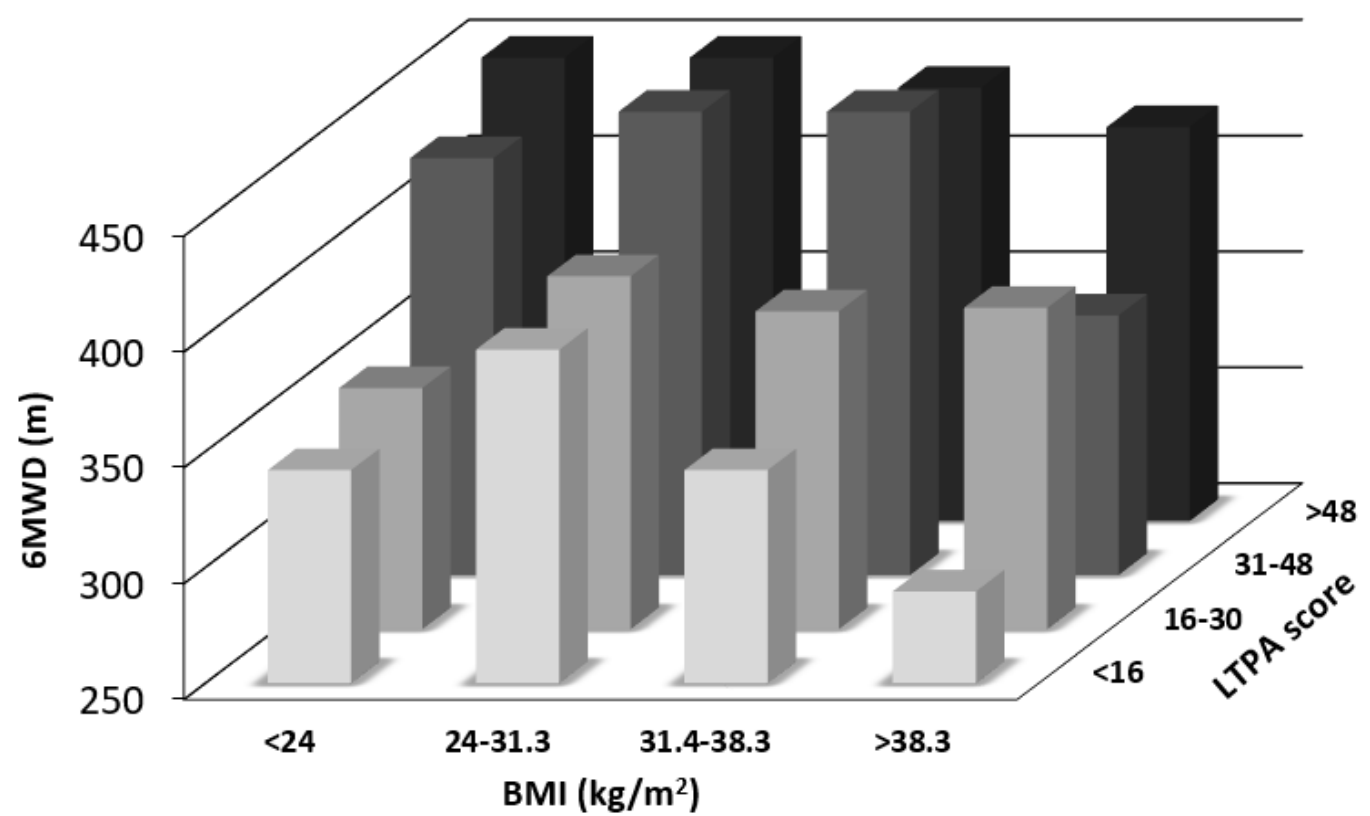

Fig. 1 Mean 6MWD (m) by LPA and BMI quartiles. LPA scores and BMI were divided into quartiles and the mean 6MWD for each quartile combination was calculated. Lower LPA scores indicate lower leisure physical activity levels. Abbreviations: $6 \mathrm{MWD}=6$ minute walk distance, $\mathrm{BMI}=$ body mass index, $\mathrm{LPA}=$ leisure physical activity.

of approximately $50 \mathrm{~m}$ is considered clinically important, indicating improved function and mobility $[37,55]$. Based on the regression results, a 1 unit decrease in BMI is associated with an increase in 6MWD of $2.6 \mathrm{~m}$. This means that BMI would have to decrease by 19 units for an improvement of $50 \mathrm{~m}$ in the 6MWD. Clearly, a 19 point decrease in BMI units is, in most cases, neither feasible nor safe or recommended. Perhaps one should not expect meaningful improvements in 6MWD or CRF based on changes in BMI. In fact, diet induced weight loss, and therefore reduced BMI, has been shown to lead to reduced CRF levels [56] and loss of fat-free mass [57]. Therefore, despite the relationship between BMI and 6MWD, weight loss by itself is not recommended as a tool to improved CRF.

While weight loss alone does not enhance CRF, it is important to consider that \%BF and $\mathrm{BMI}$ are exceedingly difficult to reduce through exercise without large amounts of training $[58,59]$. In adults with DS, a 12-week aerobic and resistance training program resulted in improvements in $\mathrm{VO}_{2}$ peak but not in BMI or skinfold thickness [17]. The null effect of exercise training on body weight in persons with DS was also supported by a meta-analysis [15]. Additionally, most weight loss is regained within 5 years [60] and weight cycling has been associated with negative health outcomes [61-63] but not always with increased mortality risk [64, 65]. However, limited evidence exists that comprehensive lifestyle interventions that incorporate PA, dieting, and other behaviour modifications may positively impact body composition in individuals with IDs [66].

While more research is needed to identify the most impactful intervention components and predictors of successful body composition change in persons with DS, diet and exercise should likely be combined to achieve weight loss and maintain or improve CRF levels. As mentioned, diet alone can lead to reduced CRF levels [56] and exercise alone may not be an effective way to reduce body weight [67]. A large amount of exercise $(700 \mathrm{kcal} /$ day $)$ without calorie compensation may be necessary to produce significant weight loss [56]. Considering the mean body weight of our participants $(73.63 \mathrm{~kg})$ and assuming a 1 MET oxygen consumption of $3.5 \mathrm{ml} / \mathrm{kg} / \mathrm{min}$ and $5.0 \mathrm{kcal} / \mathrm{L}$ 
of oxygen, our participants would have to exercise at 9 METs for about 60 minutes every day to expend 700 kcal. This would be equivalent to about 252 Godin LTPA points per week, a goal that seems out of reach for most persons with DS (the highest LTPA score in our study was 93). However, it has been shown in adults without IDs that diet and exercise combined can lead to reductions in body fat and weight while lean mass and $\mathrm{CRF}$ is maintained [56, 57]. One dietary and PA behavioral intervention study which included a child and adolescent with DS was associated with increased 6MWD while BMI and WC did not change [28].

Our results indicated that BMI may be a useful preliminary indicator of CRF and this is important because of the relationship of CRF to cardiovascular disease mortality risk and all-cause mortality risk [19, 68].

\subsection{Physical Activity and Cardiorespiratory Fitness}

Our second hypothesis that greater levels of LTPA were associated with greater CRF was supported by the results. These results are in line with another cross-sectional study comparing Special Olympics participants with DS to sedentary counterparts [26]. In our study, the correlation between LTPA and CRF was slightly stronger than the correlation between BMI and CRF (Table 2). This is also evident in Fig. 1 as 6MWD appears to increase more noticeably across quartiles of LTPA than across quartiles of BMI. The 6MWD of the participants in the fourth quartile of LTPA scores was $101 \mathrm{~m}$ farther compared to the first quartile of LTPA scores. The regression results show that a 1 unit increase in LTPA is associated with a $1.5 \mathrm{~m}$ increase in 6MWD. Thus, a 50m improvement in 6MWD would be equivalent to a 33 unit increase in LTPA (3 LTPA units are equivalent to 15 minutes of light PA, 5 LTPA units are equivalent to 15 minutes of moderate PA, and 9 LTPA units are equivalent to 15 minutes of vigorous PA). Thirty three additional units of LTPA could be achieved through various combinations of light to vigorous PA. For example, a person who engaged in an additional 56 minutes of vigorous PA per week could meet this goal. In order to improve 6MWD, a 33 unit increase in LTPA certainly seems more feasible than the aforementioned 19 point reduction in BMI. However, the actual feasibility and effectiveness of increasing LTPA by 33 units in persons with DS is unknown. Our sample had a mean of about 35 LTPA units. A 33 unit increase would mean that LTPA would have to roughly double. This goal may or may not be achievable for adults with DS.

It has been shown that PA levels can be manipulated in typical populations [69] and that increased PA levels can lead to improved $\mathrm{VO}_{2} \max$ values [25]. Among persons with DS, structured exercise interventions have been shown to improve CRF [15]. It is important to note that there is a considerable genetic component that determines CRF. In the general population, genetic factors are thought to explain about $50 \%$ of maximal oxygen uptake capacity [70]. Thus, CRF is modifiable, in part, through PA and diet in a typical population, but the extent to which this is true for people with DS is less clear. However, since both low LTPA and low CRF predict the development of metabolic syndrome [71], it is important to promote LTPA among individuals with DS as it is a modifiable risk factor.

\subsection{Physical Activity and Body Composition}

Our third hypothesis that LTPA would predict body composition was not supported. There were no significant correlations between measures of body composition or anthropometry and LTPA. In line with our results, Nordstrøm et al. [21] found no relationship between BMI and PA levels in adults with DS and other genetic disorders. This stands somewhat in contrast to an inverse relationship between PA levels and $\% \mathrm{BF}$ in 11 year old typically developing children [72]. However, the association between PA levels and $\% \mathrm{BF}$ in our study was of similar magnitude in comparison to Riddoch et al. [72], but their results may be statistically significant because of a larger sample size. Therefore, the study of the relationship between 
PA levels and body composition in individuals with DS warrants further research and we also suggest using more direct measures of PA such as DEXA to assess $\% \mathrm{BF}$.

\section{Limitations}

The primary limitation of this study is that CRF was not directly measured with a $\mathrm{VO}_{2}$ max test. However, given the high cost of metabolic testing and multiple potential barriers to measuring $\mathrm{VO}_{2} \max$ in this population, our results are clinically useful because the 6MWD correlates with direct measurements of $\mathrm{VO}_{2} \max [73,74]$ and because it can be easily administered in clinical or field settings.

It is also important to acknowledge that the $\% \mathrm{BF}$ prediction equations which the Omron BF-400 scale uses do not factor in body fat distribution and were not developed for people with DS. This is a limitation because body fat distribution may be different in people with DS compared to people without DS. However, it has been shown that bioelectrical impedance measurements can be valid in persons with DS [42]. As bioelectric impedance is a measure of body fluid and electrolyte status, hydration status should ideally be controlled. We did not control for hydration status. However, it has been shown that bioelectrical impedance measurements can be accurate regardless of hydration status [41]. Given these limitations, we recommend that the results regarding \%BF be interpreted with caution.

Another limitation is that LTPA units did not take into account that a single bout of PA could have lasted more or less than 15 minutes. Thus, LTPA units likely underestimated the true amount of LTPA because parents or guardians were supposed to report all bouts of at least 15 minutes in duration which inevitably included bouts that lasted longer and excluded bouts that lasted less than 15 minutes.

\section{Conclusion}

This study provides preliminary evidence that body composition and PA levels independently relate to CRF in young adults with DS. The nature and strength of associations between these variables needs to be further examined in order to use them in clinical health and risk assessments. A better understanding of these associations is important because persons with DS possess low CRF levels and may therefore be at increased risk for chronic conditions and functional limitations.

\section{References}

[1] Pitetti, K. H., and Campbell, K. D. 1991. "Mentally Retarded Individuals: A Population at Risk?" Medicine \& Science in Sports \& Exercise 23 (5): 586-93.

[2] Wee, S. O., Pitetti, K. H., Goulopoulou, S., Collier, S. R., Guerra, M., and Baynard, T. 2015. "Impact of Obesity and Down Syndrome on Peak Heart Rate and Aerobic Capacity in Youth and Adults." Research in Developmental Disabilities 36: 198-206.

[3] Blair, S. N., Kampert, J. B., Kohl, H. W., Barlow, C. E., Macera, C. A., Paffenbarger, R. S., and Gibbons, L. W. 1996. "Influences of Cardiorespiratory Fitness and Other Precursors on Cardiovascular Disease and All-Cause Mortality in Men and Women." Journal of the American Medical Association 276 (3): 205-10.

[4] Blair, S. N., Kohl, H. W., Barlow, C. E., Paffenbarger, R. S., Gibbons, L. W., and Macera, C. A. 1995. "Changes in Physical Fitness and All-Cause Mortality: A Prospective Study of Healthy and Unhealthy Men." Journal of the American Medical Association 273 (14): 1093-8.

[5] Blair, S. N., Kohl, H. W., Paffenbarger, R. S., Clark, D. G., Cooper, K. H., and Gibbons, L. W. 1989. "Physical Fitness and All-Cause Mortality: A Prospective Study of Healthy Men and Women." Journal of the American Medical Association 262 (17): 2395-401.

[6] Erikssen, G., Liestøl, K., Bjørnholt, J., Thaulow, E., Sandvik, L., and Erikssen, J. 1998. "Changes in Physical Fitness and Changes in Mortality." The Lancet 352 (9130): 759-62.

[7] Wei, M., Kampert, J. B., Barlow, C. E., Nichaman, M. Z., Gibbons, L. W., Paffenbarger Jr, R. S., and Blair, S. N. 1999. "Relationship between Low Cardiorespiratory Fitness and Mortality in Normal-Weight, Overweight, and Obese Men." Journal of the American Medical Association 282 (16): 1547-53.

[8] Cowley, P. M., Ploutz-Snyder, L. L., Baynard, T., Heffernan, K., Jae, S. Y., Hsu, S., and Fernhall, B. 2010. "Physical Fitness Predicts Functional Tasks in Individuals with Down Syndrome." Medicine \& Science 
in Sports \& Exercise 42 (2): 388-93.

[9] Angevaren, M., Aufdemkampe, G., Verhaar, H. J., Aleman, A., and Vanhees, L. 2008. "Physical Activity and Enhanced Fitness to Improve Cognitive Function in Older people without Known Cognitive Impairment." Cochrane Database of Systematic Reviews 3. DOI: 10.1002/14651858.CD005381.pub3.

[10] Chomitz, V. R., Slining, M. M., McGowan, R. J., Mitchell, S. E., Dawson, G. F., and Hacker, K. A. 2009. "Is There a Relationship between Physical Fitness and Academic Achievement? Positive Results from Public School Children in the Northeastern United States." Journal of School Health 79 (1): 30-7.

[11] Carmeli, E., Kessel, S., Merrick, J., and Bar-Chad, S. 2004. "A Comparison between Older Persons with Down Syndrome and a Control Group: Clinical Characteristics, Functional Status and Sensorimotor Function." Down Syndrome Research and Practice 9 (1): 17-24.

[12] Dolva, A. S., Coster, W., and Lilja, M. 2004. "Functional Performance in Children with Down Syndrome." American Journal of Occupational Therapy 58 (6): 621-9.

[13] Silverman, W. 2007. "Down Syndrome: Cognitive Phenotype." Mental Retardation and Developmental Disabilities Research Reviews 13 (3): 228-36.

[14] Andriolo, R. B., El Dib, R. P., Ramos, L., Atallah, A. N., and da Silva, E. M. 2010. "Aerobic Exercise Training Programmes for Improving Physical and Psychosocial Health in Adults with Down Syndrome." Cochrane $\begin{array}{lll}\text { Database } \quad \text { Systematic } & \text { Reviews }\end{array}$ doi:10.1002/14651858.CD005176.pub4

[15] Dodd, K. J., and Shields, N. 2005. "A Systematic Review of the Outcomes of Cardiovascular Exercise Programs for People with Down Syndrome." Archives of Physical Medicine and Rehabilitation 86 (10): 2051-8.

[16] González - Agüero, A., Vicente - Rodríguez, G., Moreno, L. A., Guerra - Balic, M., Ara, I., and Casajus, J. A. 2010. "Health-related Physical Fitness in Children and Adolescents with Down Syndrome and Response to Training." Scandinavian Journal of Medicine \& Science in Sports 20 (5): 716-24.

[17] Rimmer, J. H., Heller, T., Wang, E., and Valerio, I. 2004. "Improvements in physical fitness in adults with Down syndrome." American Journal on Intellectual and Developmental Disabilities 109 (2): 165-74.

[18] Tsimaras, V., Glagazoglou, P., Fotiadou, E., Christoulas, K., and Angelopoulou, N. 2003. "Jog-Walk Training in Cardioresperatory Fitness of Adults with Down Syndrome." Perceptual and Motor Skills 96 (3c): 1239-51.

[19] Lee, C. D., Blair, S. N., and Jackson, A. S. 1999. "Cardiorespiratory Fitness, Body Composition, and
All-Cause and Cardiovascular Disease Mortality in Men.” The American Journal of Clinical Nutrition 69 (3): 373-80.

[20] Elmahgoub, S. S., Van de Velde, A., Peersman, W., Cambier, D., and Calders, P. 2012. "Reproducibility, Validity and Predictors of Six-Minute Walk Test in Overweight and Obese Adolescents with Intellectual Disability." Disability and Rehabilitation 34: 846-51.

[21] Nordstrøm, M., Hansen, B. H., Paus, B., and Kolset, S. O. 2013. "Accelerometer-determined Physical Activity and Walking Capacity in Persons with Down Syndrome, Williams Syndrome and Prader-Willi Syndrome." Research in Developmental Disabilities 34 (10): 4395-403.

[22] Matute-Llorente, A., González-Agüero, A., Gómez-Cabello, A., Vicente-Rodríguez, G., and Casajús, J. A. 2013. "Physical Activity and Cardiorespiratory Fitness in Adolescents with Down Syndrome." Nutrición Hospitalaria 28 (4): 1151-5.

[23] Temple, V. A., Frey, G. C., and Stanish, H. I. 2006. "Physical Activity of Adults with Mental Retardation: Review and Research Needs." American Journal of Health Promotion 21 (1): 2-12.

[24] Draheim, C. C., Williams, D. P., and McCubbin, J. A. 2002. "Prevalence of Physical Inactivity and Recommended Physical Activity in Community-Based Adults with Mental Retardation." American Journal on Intellectual and Developmental Disabilities 40 (6): 436-44.

[25] Dobbins, M., Husson, H., DeCorby, K., and LaRocca, R. L. 2009. "School-Based Physical Activity Programs for Promoting Physical Activity and Fitness in Children and Adolescents Aged 6 to 18." Cochrane Database of Systematic Reviews $2 . \quad$ DOI: 10.1002/14651858.CD007651.

[26] Balic, M. G., Mateos, E. C., Blasco, C. G., and Fernhall, B. O. 2000. "Physical Fitness Levels of Physically Active and Sedentary Adults with Down Syndrome." Adapted Physical Activity Quarterly 17 (3): 310-21.

[27] Calders, P., Elmahgoub, S., de Mettelinge, T. R., Vandenbroeck, C., Dewandele, I., Rombaut, L., and Cambier, D. 2011. "Effect of Combined Exercise Training on Physical and Metabolic Fitness in Adults with Intellectual Disability: A Controlled Trial." Clinical Rehabilitation 25 (12): 1097-108.

[28] Hinckson, E. A., Dickinson, A., Water, T., Sands, M., and Penman, L. 2013. "Physical Activity, Dietary Habits and Overall Health in Overweight and Obese Children and Youth with Intellectual Disability or Autism." Research in Developmental Disabilities 34 (4): 1170-8.

[29] Li, C., Chen, S., How, Y. M., and Zhang, A. L. 2013. "Benefits of Physical Exercise Intervention on Fitness of 

Fitness Independently in Young Adults with Down Syndrome

Individuals with Down Syndrome: A Systematic Review of Randomized-Controlled Trials." International Journal of Rehabilitation Research 36 (3): 187-95.

[30] Mendonca, G. V., and Pereira, F. D. 2009. "Influence of Long-Term Exercise Training on Submaximal and Peak Aerobic Capacity and Locomotor Economy in Adult Males with Down's Syndrome." Medical Science Review 15 (2): CR33-9.

[31] Datillo, J. 1991. "Recreation and Leisure: A Review of the Literature and Recommendation for Future Directions." In Critical Issues in the Lives of People with Severe Disabilities, edited by Meyers, L., Peck, C., \& Brown, L. Baltimore, MD: Brookes.

[32] Heller, T., Hsieh, K., and Rimmer, J. 2003. "Barriers and Supports for Exercise Participation among Adults with Down Syndrome.” Journal of Gerontological Social Work 38 (1-2): 161-78.

[33] Murphy, N. A., and Carbone, P. S. 2008. "Promoting the Participation of Children with Disabilities in Sports, Recreation, and Physical Activities." Pediatrics 121 (5): 1057-61.

[34] Bertoli, M., Biasini, G., Calignano, M. T., Celani, G., De Grossi, G., Digilio, M. C., and Mazotti, S. 2011. "Needs and Challenges of Daily Life for People with Down Syndrome Residing in the City of Rome, Italy." Journal of Intellectual Disability Research 55 (8): 801-20.

[35] Lane, D. 1985. "After school: Work and Employment for Adults with Down's Syndrome?" In Current Approaches to Down's Syndrome. London, New York, Sydney, Toronto: Holt, Rinehart and Winston, 386-400.

[36] Law, M. 2002. "Participation in the Occupations of Everyday Life." American Journal of Occupational Therapy 56: 640-9.

[37] Rasekaba, T., Lee, A. L., Naughton, M. T., Williams, T. J., and Holland, A. E. 2009. "The Six-Minute Walk Test: A Useful Metric for the Cardiopulmonary Patient." Internal Medicine Journal 39 (8): 495-501.

[38] Nathan, S. D., du Bois, R. M., Albera, C., Bradford, W. Z., Costabel, U., Kartashov, A., and King, T. E. 2015. "Validation of Test Performance Characteristics and Minimal Clinically Important Difference of the 6-Minute Walk Test in Patients with Idiopathic Pulmonary Fibrosis." Respiratory Medicine 183 (7): 914-22.

[39] Amatachaya, S., Naewla, S., Srisim, K., Arrayawichanon, P., and Siritaratiwat, W. 2014. "Concurrent Validity of the 10-Meter Walk Test as Compared with the 6-Minute Walk Test in Patients with Spinal Cord Injury at Various Levels of Ability." Spinal Cord 52 (4): 333-6.

[40] Dunn, D. M., and Dunn, L. M. 2007. Peabody Picture Vocabulary Test-Fourth Edition. San Antonio, TX: Pearson.

[41] Bosy-Westphal, A., Later, W., Hitze, B., Sato, T., Kossel,
E., Glüer, C. C., and Müller, M. J. 2008. "Accuracy of Bioelectrical Impedance Consumer Devices for Measurement of Body Composition in Comparison to Whole Body Magnetic Resonance Imaging and Dual X-Ray Absorptiometry." Obesity Facts 1 (6): 319-24.

[42] Loveday, S. J., Thompson, J., and Mitchell, E. A. 2012. "Bioelectrical Impedance for Measuring Percentage Body Fat in Young Persons with Down Syndrome: Validation with Dual-Energy Absorptiometry." Acta Pcediatrica 101 (11): e491-5.

[43] Godin, G., and Shephard, R. J. 1997. "Godin Leisure-time Exercise Questionnaire." Medicine \& Science in Sports \& Exercise 29: S36.

[44] Godin, G. 2011. "The Godin-Shephard Leisure-Time Physical Activity Questionnaire." Health \& Fitness Journal of Canada 4 (1): 18-22.

[45] Casey, A. F., Wang, X., and Osterling, K. 2012. "Test-Retest Reliability of the 6-Minute Walk Test in Individuals with Down Syndrome." Archives of Physical Medicine and Rehabilitation 93 (11): 2068-74.

[46] Guerra-Balic, M., Oviedo, G. R., Javierre, C., Fortuño, J., Barnet-López, S., Niño, O., and Fernhall, B. 2015. "Reliability and Validity of the 6-min Walk Test in Adults and Seniors with Intellectual Disabilities." Research in Developmental Disabilities 47: 144-53.

[47] Nasuti, G., Stuart-Hill, L., and Temple, V. A. 2013. "The Six-Minute Walk Test for Adults with Intellectual Disability: A Study of Validity and Reliability.” Journal of Intellectual and Developmental Disability 38 (1): 31-8.

[48] Boer, P. H., and Moss, S. J. 2015. "Test-Retest Reliability and Minimal Detectable Change Scores of Twelve Functional Fitness Tests in Adults with Down Syndrome." Research in Developmental Disabilities 48: 176-85.

[49] ATS Committee on Proficiency Standards for Clinical Pulmonary Function Laboratories. 2002. "ATS Statement: Guidelines for the Six-Minute Walk Test." American Journal of Respiratory and Critical Care Medicine 166 (1): 111-7.

[50] Newman, A. B., Simonsick, E. M., Naydeck, B. L., Boudreau, R. M., Kritchevsky, S. B., Nevitt, M. C., and Harris, T. B. 2006. "Association of Long-Distance Corridor Walk Performance with Mortality, Cardiovascular Disease, Mobility Limitation, and Disability." Journal of the American Medical Association 295 (17): 2018-26.

[51] Bell, A. J., and Bhate, M. S. 1992. "Prevalence of Overweight and Obesity in Down's Syndrome and Other Mentally Handicapped Adults Living in the Community." Journal of Intellectual Disability Research 36 (4): 359-64.

[52] Rubin, S. S., Rimmer, J. H., Chicoine, B., Braddock, D., 
and McGuire, D. E. 1998. "Overweight Prevalence in Persons with Down Syndrome.” Mental Retardation 36 (3): 175-81.

[53] Okorodudu, D. O., Jumean, M. F., Montori, V. M., Romero-Corral, A., Somers, V. K., Erwin, P. J., and Lopez-Jimenez, F. 2010. "Diagnostic Performance of Body Mass Index to Identify Obesity as Defined by Body Adiposity: A Systematic Review and Meta-Analysis." International Journal of Obesity 34 (5): 791-9.

[54] Flegal, K. M., Kit, B. K., Orpana, H., and Graubard, B. I. 2013. "Association of all-cause mortality with overweight and obesity using standard body mass index categories: a systematic review and meta-analysis." JAMA 309 (1): 71-82.

[55] Perera, S., Mody, S. H., Woodman, R. C., and Studenski, S. A. 2006. "Meaningful Change and Responsiveness in Common Physical Performance Measures in Older Adults." Journal of the American Geriatrics Society 54 (5): 743-9.

[56] Ross, R., Dagnone, D., Jones, P. J., Smith, H., Paddags, A., Hudson, R., and Janssen, I. 2000. "Reduction in Obesity and Related Comorbid Conditions after Diet-Induced Weight Loss or Exercise-Induced Weight Loss in Men: A Randomized, Controlled Trial.” Annals of Internal Medicine 133 (2): 92-103.

[57] Ballor, D. L., and Poehlman, E. T. 1994. "Exercise-Training Enhances Fat-Free Mass Preservation during Diet-Induced Weight Loss: A Meta-Analytical Finding." International Journal of Obesity and Related Metabolic Disorders: Journal of the International Association for the Study of Obesity 18 (1): 35-40.

[58] Nassis, G. P., Papantakou, K., Skenderi, K., Triandafillopoulou, M., Kavouras, S. A., Yannakoulia, M., and Sidossis, L. S. 2005. "Aerobic Exercise Training Improves Insulin Sensitivity without Changes in Body Weight, Body Fat, Adiponectin, and Inflammatory Markers in Overweight and Obese Girls.” Metabolism 54 (11): 1472-9.

[59] Slentz, C. A., Duscha, B. D., Johnson, J. L., Ketchum, K., Aiken, L. B., Samsa, G. P., and Kraus, W. E. 2004. "Effects of the Amount of Exercise on Body Weight, Body Composition, and Measures of Central Obesity: STRRIDE-A Randomized Controlled Study.” Archives of Internal Medicine 164 (1): 31-9.

[60] Jeffery, R. W., Epstein, L. H., Wilson, G. T., Drewnowski, A., Stunkard, A. J., and Wing, R. R. 2000. "Long-Term Maintenance of Weight Loss: Current Status." Health Psychology 19 (1S): 5-16.

[61] Cereda, E., Malavazos, A. E., Caccialanza, R., Rondanelli, M., Fatati, G., and Barichella, M. 2011. "Weight Cycling Is Associated with Body Weight Excess and Abdominal Fat Accumulation: A Cross-Sectional Study.” Clinical
Nutrition 30 (6): 718-23.

[62] Guagnano, M. T., Ballone, E., Pace-Palitti, V., Vecchia, R. D., D’Orazio, N., Manigrasso, M. R., and Sensi, S. 2000. "Risk Factors for Hypertension in Obese Women. The Role of Weight Cycling." European Journal of Clinical Nutrition 54 (4): 356-60.

[63] Lahti - Koski, M., Männistö, S., Pietinen, P., and Vartiainen, E. 2005. "Prevalence of Weight Cycling and Its Relation to Health Indicators in Finland." Obesity Research 13 (2): 333-41.

[64] Field, A. E., Malspeis, S., and Willett, W. C. 2009. "Weight Cycling and Mortality among Middle-Aged or Older Women." Archives of Internal Medicine 169 (9): 881-6.

[65] Stevens, V. L., Jacobs, E. J., Sun, J., Patel, A. V., McCullough, M. L., Teras, L. R., and Gapstur, S. M. 2012. "Weight Cycling and Mortality in a Large Prospective US Study." American Journal of Epidemiology 175 (8): 785-92.

[66] Maïano, C., Normand, C. L., Aimé, A., and Bégarie, J. 2014. "Lifestyle Interventions Targeting Changes in Body Weight and Composition among Youth with an Intellectual Disability: A Systematic Review." Research in Developmental Disabilities 35 (8): 1914-26.

[67] Miller, W. C., Koceja, D. M., and Hamilton, E. J. 1997. "A Meta-Analysis of the Past 25 Years of Weight Loss Research Using Diet, Exercise or Diet plus Exercise Intervention." International Journal of Obesity 21 (10): 941-7.

[68] Vigen, R., Ayers, C., Willis, B., DeFina, L., and Berry, J. D. 2012. "Association of Cardiorespiratory Fitness with Total, Cardiovascular, and Noncardiovascular Mortality across 3 Decades of Follow-up in Men and Women." Circulation: Cardiovascular Quality and Outcomes 5(3): 358-64.

[69] Heath, G. W., Parra, D. C., Sarmiento, O. L., Andersen, L. B., Owen, N., Goenka, S., and Brownson, R. C. 2012. "Evidence-Based Intervention in Physical Activity: Lessons from around the World." The Lancet 380 (9838): 272-81.

[70] Bouchard, C., Daw, E. W., Rice, T., Pérusse, L., Gagnon, J., Province, M. A., and Wilmore, J. H. 1998. "Familial Resemblance for VO2max in the Sedentary State: the HERITAGE Family Study." Medicine and Science in Sports and Exercise 30 (2): 252-8.

[71] Laaksonen, D. E., Lakka, H. M., Salonen, J. T., Niskanen, L. K., Rauramaa, R., and Lakka, T. A. 2002. "Low Levels of Leisure-Time Physical Activity and Cardiorespiratory Fitness Predict Development of the Metabolic Syndrome." Diabetes Care 25 (9): 1612-8.

[72] Riddoch, C. J., Ness, A., Leary, S., Mattocks, C., Saunders, J., Deere, K., and Blair, S. 2006. "Relationship 
Fitness Independently in Young Adults with Down Syndrome

between Physical Activity and Body Composition in 11 Year Old Children." Medicine \& Science in Sports \& Exercise 38 (5): S115.

[73] Burr, J. F., Bredin, S. S., Faktor, M. D., and Warburton, D. E. 2011. "The 6-Minute Walk Test as a Predictor of Objectively Measured Aerobic Fitness in Healthy
Working-Aged Adults." Physician and Sports Medicine 39 (2): 133-9.

[74] Rikli, R. E., and Jones, C. J. 1998. "The Reliability and Validity of a 6-Minute Walk Test as a Measure of Physical Endurance in Older Adults." Journal of Aging and Physical Activity 6 (4): 363-75. 